\title{
Clinical profile of urolithiasis among hospitalized children- a single center study
}

\author{
Rajma $\mathbf{J}^{1}$, Arun $\mathrm{AC}^{2}$, Ganesapillai $\mathbf{M}^{3}$ \\ ${ }^{1}$ Dr. Jenish Rajma, Assistant Professor, Department of Pediatrics, ${ }^{2}$ Dr. Arun A.C., Assistant Professor, Department of \\ Medicine, ${ }^{3}$ Dr. Mathevan Ganesapillai, Professor, Department of Pediatrics, all authors are affiliated with Velammal \\ Medical College Hospital, Madurai, Tamil Nadu, India.
}

Address for Correspondence: Dr. Jenish Rajma, Email: jenish.rajma@ gmail.com

\begin{abstract}
Introduction: Incidence of renal and bladder stones is increasing worldwide and many children presenting with pain abdomen are diagnosed to have renal calculi. There is high rate of recurrence of stones in children if full clearance is not achieved or metabolic evaluation is not performed. We analyzed the clinical profile of the children diagnosed to have Urolithiasis and admitted in pediatric ward of our institute over the period of 2 years (Jan 2015 to Dec 2016) Methods: We conducted an Observational study done in Children $<15$ years diagnosed to have Urolithiasis based on Ultrasound findings and admitted in pediatric ward of Velammal medical college hospital, Madurai, Tamilnadu between January 2015 to December 2016. A total of 30 children were enrolled in this study and their demographic details, clinical presentation, investigations and management were analyzed. Results: Pediatric urolithiasis was more common in boys (75\%) with colicky pain abdomen (90\%) as the predominant presenting symptom. Most of the children presented during the summer months. They had associated complications like urinary tract infection, hydronephrosis and Pelviureteric junction obstruction. Ureter was the commonest site of urolithiasis (18/30) and most of the children responded to medical management. The children who presented with recurrent symptoms during this study period were evaluated and diagnosed to have some underlying metabolic or genetic disorder. Conclusion: Rising incidence of renal stones with acute colicky pain in pediatric age group is a worrisome problem as it causes school absenteeism and mental stress to the entire family. Urolithiasis in children needs complete evaluation to rule out underlying metabolic disorder. Complete evaluation of the underlying etiology is essential to prevent recurrence and further complications.
\end{abstract}

Keywords: Kidney calculi, Urinary bladder calculi, Obesity

\section{Introduction}

Incidence of renal and bladder stones is increasing worldwide and especially many children presenting with pain abdomen are diagnosed to have renal calculi $(1,2)$. The changing dietary habits and increasing prevalence of obesity is a major risk factor in children. Two important risk factors for stone formation in children are not drinking enough fluids and eating too much salt; both increase the amount of calcium and oxalate in the urine. There is high rate of recurrence of stones in children if full clearance is not achieved or metabolic evaluation is not performed and managed $(3,4)$. There is significant change in the clinical profile of pediatric urolithiasis in developing nations. Bladder

Manuscript received: $6^{\text {th }}$ May 2017

Reviewed: $16^{\text {th }}$ May 2017

Author Corrected: $24^{\text {th }}$ May 2017

Accepted for Publication: $31^{\text {st }}$ May 2017 stones have become rare (5) and many asymptomatic obese children (6) are diagnosed to have renal calculi as a part of the metabolic syndrome. The widespread availability of Ultrasound is also an important reason for increasing diagnosis of urolithiasis. We analyzed the clinical profile of the children diagnosed to have Urolithiasis and admitted in pediatric ward of our institute over the period of 2 years (Jan 2015 to Dec 2016)

\section{Materials and Methods}

Objective of the study: To study the clinical manifestations, management and outcome of all children diagnosed as urolithiasis and analyze the factors associated with recurrent episodes of renal stone formation. 
Place and duration of study: Velammal medical college hospital, Madurai, Tamilnadu, January 2015 to December 2016

Study design: Observational study

Study population: Children $<15$ years diagnosed to have Urolithiasis based on Ultrasound findings
The study was conducted after getting ethical committee clearance from the institute. Data collection was done by reviewing the case sheets from medical records department and contacting the patient over phone to collect additional details. Data was entered in Microsoft excel and analysis was done.

\section{Results}

Out of the 30 cases, almost three fourth of the cases were boys. About 23 out of the total 30 children (77\%) were boys, showing a significant male predominance (Fig 1), the sex predilection more evident after 6 years of age. 5 of them had a positive family history of renal calculi.3 of them had recurrent renal calculi requiring multiple hospitalizations and repeated surgical intervention. 6 children were less than 5 years of age, 13 children were between 6 to 10 years while 11 of them were between 11 to 15 years.

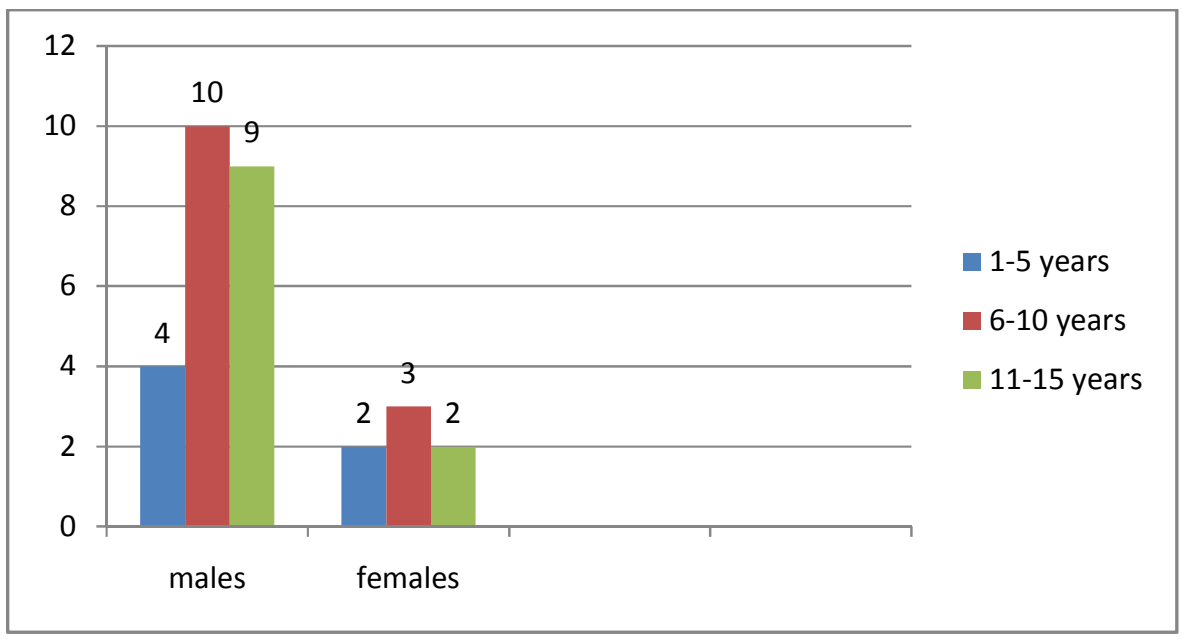

Figure-1: Pediatric Urolithiasis: age distribution.

Almost $90 \%$ of them had pain abdomen as the predominant symptom which was colicky and radiating. Around 53\% had associated vomiting, while 33\% had associated urinary tract infection like symptoms (10/30) (Table 1). History of similar illness in the past was present in nine children. Few children (3/30) presented with anuria while in ultrasound 8 had features of hydronephrosis secondary to obstruction commonly in the pelviureteric junction

Table-1: Pediatric Urolithiasis: Symptomatology.

\begin{tabular}{|c|c|c|}
\hline Symptoms & No. of children & Percentage \\
\hline Pain abdomen & $27 / 30$ & $90 \%$ \\
\hline Vomiting & $16 / 30$ & $53 \%$ \\
\hline Oligoanuria & $3 / 30$ & $10 \%$ \\
\hline Hematuria & $3 / 30$ & $33 \%$ \\
\hline UTI like illness & $10 / 30$ & $30 \%$ \\
\hline Previous similar episodes & $9 / 30$ & $16.6 \%$ \\
\hline Positive family history & $5 / 30$ & $10 \%$ \\
\hline
\end{tabular}

The maximum number of children presenting with a renal colic due to calculi was in summer months (May, June) and mild increase in cases during October, November, December which is predominantly rainy in this part of the country (Fig 2). 


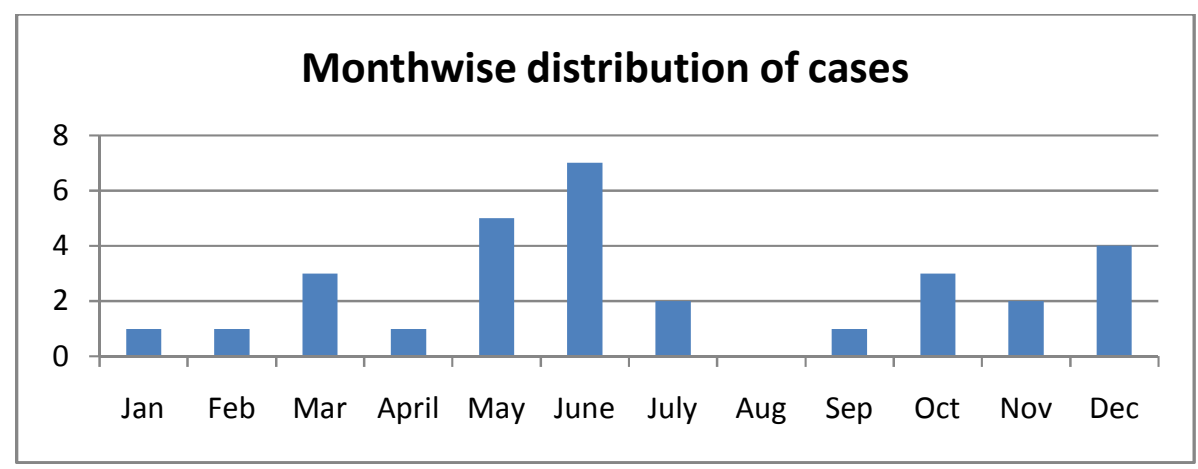

Figure-2: Pediatric urolithiasis- Month wise Case distribution

The diagnosis of urolithiasis was made based upon Ultrasound findings- to identify the site and size of calculi. 18 children had ureteric calculi (Fig 3) which was the commonest and symptomatic, of which 5 had associated renal calculi. On further analysis among the different age groups, the most common site of renal calculi was in the ureter. Out of the 11 children in the 11 to 15 year age group, 8 of them had ureteric calculi while 2 of them had both renal and ureteric calculi and 1 had both renal and vesical calculus. Out of the 13 children in 6 to 10 age group, 7 had ureteric and 6 had renal calculi. Only 6 children were in the 1 to 5 age group, with 3 ureteric and 2 renal calculi. Bladder calculi were present in 4 children more than 5 years of age.

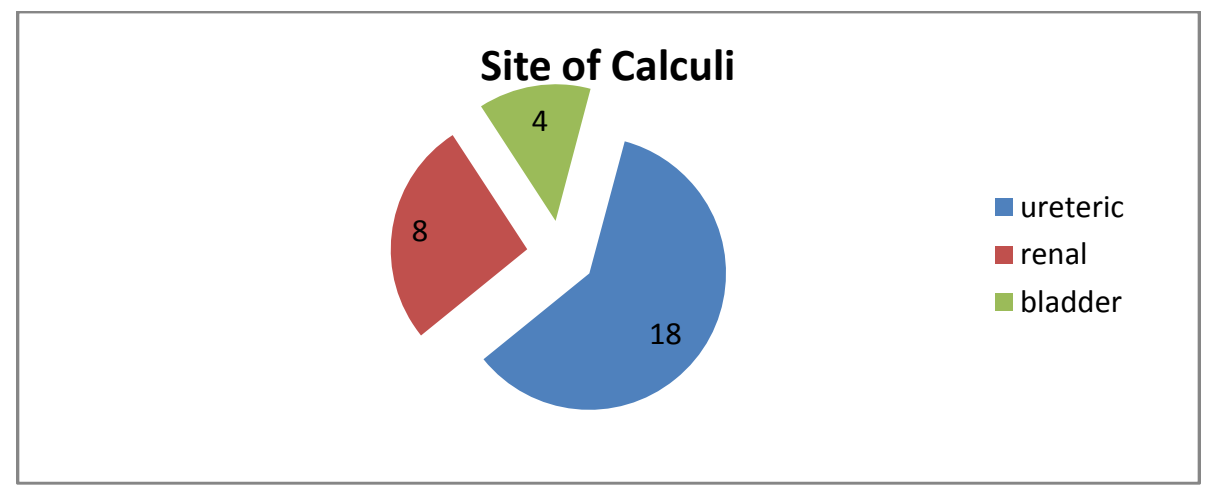

Figure-3: Pediatric Urolithiasis- Site of Calculi.

Out of the total 30 children enrolled in the study, 18 children had spontaneous passage of the calculi with conservative medical management while 12 of them required surgery. Most of the children (14/18) had symptomatic relief with analgesics and hydration. They had spontaneous passage of the renal stones within 48-72 hours. They were discharged within 5 days. Few children (4/18) required oral tamsulosin for 3 days.

Evidence of associated urinary tract infection in the form of pyuria, bacteriuria and trace proteinuria were present in 17 out of the 30 children and 3 of them had culture positivity- significant colonies of E. coli(2/3) and proteus (1/3) had grown in urine culture. Hence they were treated for the same. Others who were symptomatic but negative cultures were given empiric oral antibiotics.

Some children presented with associated complications of calculi like Hydroureteronephrosis (6/10) and pelviureteric junction obstruction (4/10). One child had pyonephrosis with E.coli grown in culture requiring i.v antibiotics followed by percutaneous nephrolithotomy. Spontaneous passage of stone was observed in 4 children with hydroureteronephrosis with medical management. Repeat ultrasound done after 5 days showed normal kidneys and ureters. 2 children required surgical intervention. The need for surgical intervention was more in the younger age groups. Between 1-5 years, 4 out of 6 required surgery while among children between 11 to 15 years only 4 out of 11 required surgical intervention. The most common surgical intervention done was percutaneous nephrolithotomy (6/12), 2 children were treated with extracorporeal shockwave lithotripsy and stenting. Out of the 4 children with bladder stones, 3 were treated with open cystolithotomy while one was treated cystoscopically. 


\section{Discussion}

Urolithiasis in the pediatric population is an important cause of morbidity worldwide [1,2,3,4]. Presenting signs and symptoms are often considerably different from those in adults. Kidney stone is not a disease itself, but it is only a symptom. Therefore, its early diagnosis is mandatory for every child with the first stone event. Vague abdominal pain, hematuria, and urinary tract infection are more common in children than the classic colicky flank pain [1]. It has become more common in children over the past few decades as a result of rapid variations in food habits and increasing affluence.

Changing socio-economic conditions have generated changes in the incidence and type of urolithiasis in terms of both the site and the physico-chemical composition of the calculi. Up to $50 \%$ of the children may have underlying abnormalities leading to formation of kidney stones such as high calcium in urine, low citrate in urine, high uric acid or oxalates in urine [2]. A study published in 1991 from Western India over duration of 9 years had a total of 77 children with $6.4 \%$ incidence in children from the overall total urolithiasis cases [5]. This shows a significant increase in the prevalence of urolithiasis in children over the last twenty years.

Childhood obesity [6] is also a risk factor for stone formation. Children today seem to live a lifestyle that puts them at risk of kidney stones, meaning they consume sugar-filled drinks and a fast-food diet that is high in sodium, a known risk factor in the formation of kidney stones. Extensive epidemiological observations have emphasized the importance of nutritional factors in the pathogenesis of urolithiasis, and specific dietary advice is, nowadays, often the most appropriate for prevention and treatment of urolithiasis. A familial occurrence has also been suggested for hypercalciuria, one of the main risk factors for idiopathic urolithiasis.

There are few important epidemiologic variations in Urolithiasis presentation between developed and developing countries. In our study, there was a significant male predominance, which was similar to other Asian studies [7,8 ] with 2:1 to 3:1 ratio. But some studies in children [5] did not find significant male predominance in children than in adults. Also the most common site of calculi is in ureter or renal pelvis. A report from Pakistan demonstrated a shift in the predominant location of urinary calculi from the bladder to the upper urinary tract over a 13-year period from
1987 to 2000 [9]. In some older studies from India also [5], urinary bladder calculi (67.5\%) were more common than upper urinary tract but currently the scenario is changing and getting similar to the developed nations.

The seasonal increases in cases were noticed during summer months which could have been due to dehydration coupled with decreased fluid intake. UTI is also another common presenting sign of urolithiasis in children. In a study around 54\% of children referred with UTI had calculi [5]. Urinary tract infection is an important disease, which can highlight the presence of renal stone diseases. In this respect, clinicians should take a precise history regarding urinary tract infection and ask for urine analysis and urine culture.

In the adolescent boys, 3 out of the 9 were obese with body mass index more than 22 . In the other age groups we did not find any significant metabolic syndrome associated with obesity and renal stones in this study. In those who presented with recurrent urolithiasis, hypercalciuria was noticed in 2 children, others had normal 24 hour calcium creatinine ratio. Stone analysis was done in 8 of them, with most of them turning out to be calcium oxalate $[9,11]$, one struvite stone and one rare case of silica stone [10] was also identified. Silicate stones are extremely rare type of urolithiasis, with incidence of $0.2 \%$ in humans. It is usually associated with consumption of a large amount of silicate containing antacids such as magnesium trisilicate. Other risk factors for silicate stone are malnutrition, infection, diet poor in proteins, drugs such as zonisamide (antiepileptic). In a sub-saharan study [11], Stone composition in urolithiasis in children was a heterogeneous disorder, with hyperuricosuria, insufficient diuresis, and infection due to malnutrition seems to be the common causes. There has been a change in the pattern of stone composition with an increase in the frequency of kidney stones and a decrease in bladder stones.

The role of familial predisposition and environmental factors in pediatric urolithiasis is now better understood. Metabolic factors are more common in pediatric urolithiasis than in adult stone disease [3, 12]. One 10 year old boy who presented with recurrent renal calculi and short stature along with cataract in both eyes was diagnosed as Dent's disease. He required surgical interventions in the form of percutaneous nephrolithotomy due to recurrent renal stones in both 
kidneys which were more than $8 \mathrm{~mm}$ in size. A three year old who presented with renal stones, gall stones and hepatomegaly was diagnosed to have glycogen storage disease type 1 . One adolescent boy who presented with ureteric calculi was a known Type 1 Diabetes mellitus on regular insulin therapy. There is increased risk of underlying metabolic or genetic disorders in children presenting at a very young age or with associated other manifestations.

An European retrospective study [13] between 2009 and 2013 of 68 children ( 34 boys and 34 girls) under 3 years revealed risk factors like metabolic disorders (up to $80 \%)$, infections of the urinary tract $(46 \%)$, urinary system malformations (10-20\%). Among the metabolic disorders, the most common are hypercalciuria (34\%), hyperuricosuria $(8 \%)$, cystinuria $(7 \%)$ were identified in many studies [14-16]. Dietary management gives significant results if the underlying metabolic cause is identified [18]. In recent publications, it is recommended to begin the treatment with the high daily fluid intake and dietary alterations consistent with the metabolic disorders, a low-sodium diet for hypercalciuria, and oligopurine diet for hyperoxaluria. But there are no similar large scale Indian studies for complete evaluation of underlying metabolic or biochemical abnormality.

The recurrence rate of nephrolithiasis in patients with kidney stones that are developed during childhood is poorly defined $[3,4]$. Within ten years, this disease usually recurs in more than $50 \%$ of patient. Metabolic factors are determined by the assessment of urinary solutes and naturally occurring inhibitors of crystal and stone formation such as citrate. Lack of widespread availability of urinalysis to identify hypocitraturia or hyperuricosuria in our setting was an important setback.

Urine Analysis should be deferred until UTI has been treated completely and at least four weeks after lithotripsy or resolution of the obstruction. The normal values for the excretion rates of solutes are affected by regional and cultural variability.

Many children and adolescents with stones have more than one predisposing factor. When an inherited metabolic disorder is suspected, urine samples from family members can be help to diagnosis. In patients with multiple stones at the onset, or those in whom there is active stone formation and no anatomical abnormalities have been determined, additional examinations can be helpful [19].
Most commonly used diagnostic modality in our study was Ultrasound, as in many other studies. Although ultrasound is less sensitive than CT, ultrasound is usually a good initial choice and the most suitable method for the detection of urolithiasis in children because of radiation absence, no requirement of anesthesia, providing detailed anatomic information, cost-benefit issues, wide availability, detection of hydronephrosis and other anatomical disorders of the urinary tract.

The success of this imaging method depends on the observer's skills [20]. Non-contrast spiral computed tomography (CT) scan is the gold standard for the diagnosis of nephrolithiasis, because it has an excellent visualization of all stones whether they are opaque or nonopaque. Except for indinavir stones, size and location of stones, it is rapid and nearly $100 \%$ sensitive and specific. CT scan provides detailed anatomic information and is sensitivity for very small stones. It is recommended to perform CT scan in children with refractory urolithiasis symptoms but without clear evidence of stone in KUB and ultrasonography [19].

Medical explosive therapy includes the drugs that expel ureteral stones by relaxing smooth muscle. Calcium channel and alpha-1 receptors blockers are the main prescribed drugs. In some guidelines, this therapy is not recommended, because of little data in pediatric age group. Some clinicians administer alpha blocker medications in children with distal ureteral stone. We tried tamsulosin in 3 children with ureterovesical junction stones which had good response in all 3 children. None of them required surgical intervention. But the dose and duration for pediatric cases has not been well proven by trials. Most of the children enrolled in this study were given only hydration- oral and intravenous, along with analgesics.

Percutaneous nephrolithotomy (PCNL) is a less invasive treatment compared with open surgery. Therefore, this technique is a useful alternative to open surgery in children. The indications of PCNL are similar in children and adults. PCNL is indicated in renal stones with a diameter more than $20 \mathrm{~mm}$. Most pediatric urinary tract stones are removed by ESWL, ureteroscopy and PCNL and a low number of stones are removed by open surgery.

The indication of open surgery is the presence of large stones especially in small children and very obese children and those with malformation 


\section{Conclusion}

Rising incidence of renal stones with acute colicky pain in pediatric age group is a worrisome problem as it causes school absenteeism and mental stress to the entire family.

Urolithiasis in children needs complete evaluation to rule out underlying metabolic disorder. Parents need to be counseled on proper dietary modifications in order to prevent recurrence and further morbidities.

Complete evaluation of the underlying etiology is essential to prevent recurrence and further complications. Long term follow up of this cohort for recurrence of calculi and epidemiologic associations will help to identify the unknown and unclear etiologies behind the increasing incidence of renal calculi in the present generation of Indian population.

\section{Abbreviations}

Percutaneous nephrolithotomy PCNL, Extracorporeal shockwave lithotripsy ESWL, Pelviureteric junction PUJ computed tomography CT, Urinary tract infection UTI.

Funding: Nil, Conflict of interest: None initiated, Perission from IRB: Yes

\section{References}

1. Kokorowski PJ, Hubert K, Nelson CP. Evaluation of pediatric nephrolithiasis. Indian J Urol. 2010;26:531-5. doi: 10.4103/0970-1591.74453.

2. D avid J. Sas. An Update on the Changing Epidemiology and Metabolic Risk Factors in Pediatric Kidney Stone Disease CJASN August 20116 ): (8) 2062-2068; doi:10.2215/CJN.11191210

3. Akhavan Sepahi M, Heidari A, Shajari A. Clinical manifestations and etiology of renal stones in children less than 14 years age. Saudi J Kidney Dis Transpl 2010; 21(1): 181-4

4. Valentini RP, Lakshmanan Y. Nephrolithiasis in Children. Adv Chron Kidney Dis 2011; 18 (5): 370-5.

5. Shah, A.M., Kalmunkar, S., Punekar, S.V. et al. Spectrum of pediatric urolithiasis in western India. Indian J Pediatr (1991) 58: 543. doi: 10.1007/ BF02750939.
6. Hernandez JD, Ellison JS, Lendvay TS. Current Trends, Evaluation, and Management of Pediatric Nephrolithiasis. JAMA Pediatr. 2015;169(10):964-970. doi:10.1001/jamapediatrics.2015.1419

7. Aamer Mehmood Khan, Muhammad Shahid Hussain, Khemchand N Moorani*, Khalid Mehmood Khan Urolithiasis Associated Morbidity in Children. Journal of Rawalpindi Medical College (JRMC); 2014; 18(1): 73-74

8. Javed Altaf, Adeel Hyder Arain, Nand Lal Kella, Shafique-ur-Rehman. Chemical Analysis of Urinary Stones and its Locations Associated to Urinary Tract. J Liaquat Uni Med Health Sci. 2013; 12(3): 203-207.

9. Rizvi SA, Naqvi SA, Hussain Z, Hashmi A, Hussain M, Zafar MN. et al. Pediatric urolithiasis: developing nation perspectives. J Urol. 2002;168:1522-5. doi: 10.1097/01.ju.0000028601.63446.51

10. Pichandi Rajarajan, Raamya Sathish Kumar Motilal Silicate urolithiasis in a paediatric patient; Indian journal of Urology; 2017; 33(2): 165-166

11. Angwafo III F.F. ${ }^{\mathrm{a}}{ }^{\mathrm{d}}$. Daudon M. $^{\mathrm{b}}$. Wonkam A. ${ }^{\mathrm{a}} \cdot$ Kuwong P.M. ${ }^{\mathrm{c}} \cdot$ Kropp K.A. Pediatric Urolithiasis in Sub-Saharan Africa: A Comparative Study in Two Regions of Cameroon.Eur Urol 2000;37:106-111 (DOI:10.1159/000020109).

12. Sharma AP, Filler G. Epidemiology of pediatric urolithiasis. Indian Journal of Urology : IJU : Journal of the Urological Society of India. 2010;26(4):516-522. doi:10.4103/0970-1591.74450.

13. Aaslid A., Tkaczyk M. The clinical course urolithiasis children under 3 years Prog Health Sci 2014, 4(1):68-74

14. Sarkissian A, Babloyan A, Arikyants N, Hesse A, Blau N, Leumann E. Pediatric urolithiasis in Armenia: a study of 198 patients observed from 1991 to 1999. Pediatr Nephrol. 2001 Sep;16(9):728-32.

15. Alpay H, Ozen A, Gokce I, Biyikli N. Clinical and metabolic features of urolithiasis and microlithiasis in children. Pediatr Nephrol. 2009 Nov;24(11):2203-9.

16. Sarica K. Pediatric urolithiasis: etiology, specific pathogenesis and medical treatment. Urol Res. 2006 Apr;34(2):96-101. 
17. Acar B, Inci Arikan F, Emeksiz S, Dallar Y. Risk factors for nephrolithiasis in children. World $\mathrm{J}$ Urol. 2008 26:627-30.

18. Baştuğ F, Düşünsel R. Pediatric urolithiasis: causative factors, diagnosis and medical management. Nat Rev Urol. 2012 Feb 7;9(3):138-46.
19. Akhavan Sepahi M, Hosini R. Approach and Medical Management of Urinary Tract Stone in Children. Caspian J Pediatr March 2016; 2(1): 86-95.

20. Fowler KA, Locken JA, Duchesne JH, Williamson MR. US for detecting renal calculi with nonenhanced CT as a reference standard. Radiology 2002; 222(1): 109-13.

\section{How to cite this article?}

Rajma J, Arun AC, Ganesapillai M. Clinical profile of urolithiasis among hospitalized children- a single center study. J PediatrRes.2017;4(05):343-349.doi:10. 17511/ijpr.2017.i05.09 